\title{
Functional Interplay between Posterior Parietal and Ipsilateral Motor Cortex Revealed by Twin-Coil Transcranial Magnetic Stimulation during Reach Planning toward Contralateral Space
}

\author{
Giacomo Koch, ${ }^{1,2,3}$ Miguel Fernandez Del Olmo, ${ }^{1,4}$ Binith Cheeran, ${ }^{1}$ Sven Schippling, ${ }^{1}$ Carlo Caltagirone, ${ }^{2,3}$ Jon Driver, ${ }^{5,6}$ \\ and John C. Rothwell ${ }^{1}$ \\ ${ }^{1}$ Sobell Department of Motor Neuroscience and Movement Disorders, Institute of Neurology, University College London, London WC1N 3BG, United \\ Kingdom, ${ }^{2}$ Laboratorio di Neurologia Clinica e Comportamentale, Fondazione Santa Lucia, Instituto di Ricovero e Cura a Carattere Scientifico, 00179 Rome, \\ Italy, ${ }^{3}$ Clinica Neurologica, Dipartimento di Neuroscienze, Università di Roma Tor Vergata, 00133 Rome, Italy, ${ }^{4}$ Instituto Nacional de Educación Física, \\ Galicia, 15179 La Coruña, Spain, and ${ }^{5}$ Institute of Cognitive Neuroscience and ${ }^{6}$ Department of Psychology, University College London, London WC1N 3AR, \\ United Kingdom
}

Posterior parietal cortex (PPC) has connections with motor and premotor cortex, thought to transfer information relevant for planning movements in space. We used twin-coil transcranial magnetic stimulation (tcTMS) methods to show that the functional interplay between human right PPC and ipsilateral motor cortex (M1) varies with current motor plans. tcTMS during the reaction time of a reach task revealed facilitatory influences of right PPC on right M1 only when planning a (contralateral) leftward rather than rightward reach, at two specific time intervals ( 50 and $125 \mathrm{~ms}$ ) after an auditory cue. The earlier reach-direction-specific facilitatory influence from PPC on M1 occurred when subjects were blindfolded or when the targets were presented briefly, so that visual feedback corrections could not occur. PPC-M1 interplay was similar within the left hemisphere but was specific to (contralateral) rightward planned reaches, with peaks at 50 and $100 \mathrm{~ms}$. Functional interplay between human parietal and motor cortex is enhanced during early stages of planning a reach in the contralateral direction.

Key words: transcranial magnetic stimulation; connectivity; reaching; parietal cortex; motor control; motor-evoked potential

\section{Introduction}

Posterior parietal cortex (PPC) may be involved in transforming information about the location of targets in space, into signals more related to motor intentions, such as reaching to that point (Mountcastle et al., 1975; Kalaska et al., 1990; Kalaska and Crammond, 1995; Lacquaniti et al.,1995; Mountcastle, 1995; Caminiti et al., 1996; Johnson et al., 1996; Battaglia-Mayer et al., 2000, 2003; Andersen and Buneo, 2002; Buneo et al., 2002; Cohen and Andersen, 2002). PPC may transfer such information to premotor and motor cortices via white-matter tracts of the superior longitudinal fasciculus (Tanne-Gariepy et al., 2002; Croxson et al., 2005; Makris et al., 2005; Rushworth et al., 2006; Rozzi et al., 2006), but a motor role for PPC has been debated, with alternative views suggesting that PPC may represent the currently at-

Received Dec. 19, 2007; revised April 5, 2008; accepted April 8, 2008.

J.D. was supported by the Wellcome Trust and holds a Royal Society-Leverhulme Trust Senior Research Fellowship. J.C.R. is supported by the Wellcome Trust and Medical Research Council (United Kingdom). We thank the anonymous reviewers for their helpful comments.

Correspondence should be addressed to Prof. John C. Rothwell, Sobell Department of Motor Neuroscience and Movement Disorders, Institute of Neurology, UCL, Queen Square, London WC1N 3BG, UK. E-mail: j.rothwell@ion.ucl.ac.uk.

DOI:10.1523/JNEUROSCI.0957-08.2008

Copyright $\odot 2008$ Society for Neuroscience $\quad$ 0270-6474/08/285944-10\$15.00/0 tended or most salient stimulus location rather than nascent motor plans per se (Colby and Goldberg, 1999).

Recently we developed (Koch et al., 2007a) a new method for studying functional "connections," or functional interplay, between PPC to ipsilateral motor cortex non-invasively in humans, using a "paired-pulse" or twin-coil transcranial magnetic stimulation (tcTMS) paradigm. A conditioning TMS pulse is applied over PPC, shortly before a test pulse over the hand area of motor cortex (M1). The latter pulse evokes a small twitch in contralateral hand muscles, measurable with surface electromyography (EMG). When the interval between the PPC and M1 pulses is $\sim 4-6 \mathrm{~ms}$, the EMG response triggered by the M1 pulse is enhanced (Koch et al., 2007a), indicating that the PPC pulse has altered excitability of M1, consistent with a functional PPC-M1 connectivity or interplay. The site of the conditioning PPC pulse leading to the most pronounced impact on M1 lay over the caudal intraparietal sulcus (cIPS) (Koch et al., 2007a). Similar tcTMS designs had been used previously to reveal functional effects between motor cortex with contralateral M1 (Ferbert et al., 1992) or premotor cortex (PMd) (Civardi et al., 2001; Mochizuki et al., 2004; Baumer et al., 2006; Koch et al., 2006, 2007b). An advantage of the tcTMS approach is temporal precision. The effect of a conditioning TMS pulse on the response to the M1 test pulse 
must presumably reflect excitability of functional connections at the time TMS is given, and this excitability might vary with the current motor or task state. Thus, if PPC can deliver information related to, say, the target location for an upcoming arm movement, then excitability of the inferred $\mathrm{PPC}-\mathrm{M} 1_{\text {hand }}$ connection may change before movement onset and might relate specifically to planned reaches in the contralateral direction (Mattingley et al., 1998). If instead PPC merely represents currently salient or task-relevant locations rather than providing information for specific reaching plans, then the impact of PPC TMS on the M1 response in a tcTMS paradigm should not change with motor context (Snyder et al., 1997). The present experiment tested this for the first time in humans, examining how the recently described tcTMS effects between human PPC and M1 (Koch et al., 2007a), that had initially been discovered at rest, might vary with motor context.

\section{Materials and Methods \\ Subjects}

Fifteen healthy volunteers (eight men and seven women, 21-34 years old) participated in the various experiments. All were right-handed based on the Edinburgh Handedness Inventory. Written informed consent was obtained in accord with local ethics.

\section{Experiment 1}

Experimental procedure. We used an auditory choice reaction time (RT) task similar to Koch et al. (2006) but adapted it to emphasize directional reaching in accord with our hypotheses about PPC-M1 influences. Subjects $(n=10)$ sat on a 45 -cm-high straight-back chair facing a table, 120 $\mathrm{cm}$ wide $\times 60 \mathrm{~cm}$ deep, placed at a height of $70 \mathrm{~cm}$. On the opposite edge of the table was fixed an upright, flat wooden panel, $80 \mathrm{~cm}$ wide $\times 50 \mathrm{~cm}$ high, placed at $60 \mathrm{~cm}$ distance from the subject. A headrest ensured a constant head position throughout the experiment. During target presentation the room was illuminated with direct lighting from the ceiling. The upright panel and the top of the table were painted white. Subjects placed the index finger of their left hand on an upraised bump (2.5 $\mathrm{cm}$-diameter coin) on the table surface that could be located by touch. This point was in line with their sagittal midline. The peripheral targets comprised 2-cm-diameter blue circles, subtending $0.5^{\circ}$, positioned 20 $\mathrm{cm}$ left or right of a fixation cross sized $2 \mathrm{~cm}$ at a viewing distance of 60 $\mathrm{cm}$. The distance between the hand at its start position and the more distant fixation point was $30 \mathrm{~cm}$ (supplemental figure, available at www.jneurosci.org as supplemental material).

Subjects were required to reach to and touch the peripheral left or right target as soon as they heard a cue sound. Each trial began with an auditory warning $(500 \mathrm{~Hz}, 40 \mathrm{~ms})$. After this sound, subjects had to fixate the fixation cross in the middle of the panel. Eye position was assessed trough electrooculogram (EOG). The imperative auditory signal was given randomly 1-3 s later and consisted of either a high $(800 \mathrm{~Hz}, 30 \mathrm{~ms})$ or low $(200 \mathrm{~Hz}, 30 \mathrm{~ms})$ frequency tone pulse that indicated which peripheral target to reach toward (and hence whether to reach in the left or right direction), according to counterbalanced instructions given to the subjects (high meaning reach right, low meaning reach left, or vice versa, fully counterbalanced as described below). The intertrial interval was $6 \mathrm{~s}$. At the start of each block of trials, the high and low tones were assigned randomly to indicate that subjects had to reach correspondingly to the right or left target; these instructions were counterbalanced within and across subjects. The order of different experimental blocks was counterbalanced across subjects. Before each test session, at least 10 practice trials were given until at least five continuous RTs were within $150 \%$ of mean RT.

Transcranial magnetic stimulation. EMG traces were recorded from the first dorsal interosseous (FDI) muscles of the left hand using 9-mmdiameter, $\mathrm{Ag} / \mathrm{AgCl}$ surface cup electrodes. Although the transport component of a reach movement depends on the activity of muscles acting at the shoulder joint, we choose to test the FDI because this muscle was selectively activated in our reaching to point task and motor-evoked potentials (MEPs) can be recorded more reliably from this muscle com- pared with proximal shoulder muscles. The active electrode was placed over the muscle belly and the reference electrode over the metacarpophalangeal joint of the index finger. Responses were amplified with a Digitimer D360 amplifier through filters set at $20 \mathrm{~Hz}$ and $2 \mathrm{kHz}$ with a sampling rate of $5 \mathrm{kHz}$ and then recorded by a computer using SIGNAL software (Cambridge Electronic Devices). We used a paired-pulse stimulation technique with two high-power Magstim 200 machines. The magnetic stimulus had a nearly monophasic pulse configuration with a rise time of $\sim 100 \mu \mathrm{s}$, decaying back to 0 over $\sim 0.8 \mathrm{~ms}$.

First the intensity of TMS was adjusted to evoke an MEP of $\sim 1 \mathrm{mV}$ peak to peak in the relaxed left FDI. The hand motor area of right M1 was defined as the point at which stimulation evoked the largest MEP from the contralateral FDI muscle. The test stimulator was connected to a small custom-made figure-of-eight-shaped coil (external diameter, 50 $\mathrm{mm}$ ). The coil over M1 was always placed tangentially to the scalp at $45^{\circ}$ angle from the midline of the central sulcus, inducing a posteroanterior current flow.

The conditioning stimulator was connected to a normal figure-ofeight-shaped coil (external diameter, $70 \mathrm{~mm}$ ). The coil position for right PPC TMS was defined relative to the P4 position of the 10-20 EEG system. According to previous investigations adopting threedimensional magnetic resonance imaging (MRI) reconstruction, this site is situated close over a part of the angular gyrus (AG) in the inferior parietal lobule (IPL) and close to a posterior part of the adjoining intraparietal sulcus (cIPS) (Herwig et al., 2003; Rushworth and Taylor, 2006; Koch et al., 2007a). The center of the coil was positioned over P4 tangentially to the skull, with the handle pointing downward and slightly medial $\left(10^{\circ}\right)$ to induce a posteroanterior directed current in the underlying cortical tissue. In three representative subjects, MRI guided frameless stereotaxy (Brainsight Frameless; Rogre Research) was used to verify the trajectory of the induced magnetic field and the distance from the underlying portion of cortex in respect to $\mathrm{P} 4$ position (Fig. $1 \mathrm{~A}$ ).

EOG responses were recorded by means of $\mathrm{Ag} / \mathrm{AgCl}$ surface electrodes producing binocular horizontal EOG. The signal was amplified and bandpass filtered $(5-2000 \mathrm{~Hz})$ using SIGNAL software. The upward deflection of the recorded eye position signal in EOG channels corresponded to rightward eye movement. EOG was used to assess saccades and initial saccadic latencies in experiment 3 (see below).

TMS was delivered over M1 of the right hemisphere at different delays $(25,50,75,100,125$, and $150 \mathrm{~ms})$ after the cue sound. On the basis of our recent published paired-pulse TMS work with PPC and M1 sites (Koch et al., 2007a), we used the following parameters to best activate PPC-M1 interplay. The intensity of the M1 pulse was adjusted to evoke an MEP of $\sim 1 \mathrm{mV}$ peak to peak in the relaxed left FDI. In half of the trials, M1 TMS was preceded by a conditioning pulse delivered $4 \mathrm{~ms}$ earlier, over the ipsilateral PPC at an intensity of $90 \%$ of the resting motor threshold (RMT). We defined RMT as the lowest intensity that evoked five small responses $(\sim 50 \mu \mathrm{V})$ in the contralateral FDI muscle in a series of 10 stimuli when the subject kept the FDI muscles relaxed in both hands.

Ten trials were performed for each subcondition in the main experiment (left or right movement planned, as instructed by the symbolic auditory imperative stimulus, fully crossed with M1 pulse alone or paired PPC-M1 pulses), at two delays selected pseudorandomly in each block for each subject. Furthermore, in each session, any "baseline" conditioning effects of right PPC on ipsilateral M1 were measured at rest in a separate block. This block comprised 20 trials in which a PPC pulse preceded an M1 pulse for half of the trials. EMG was measured on each individual trial. The mean peak-to-peak amplitude of the conditioned MEP was expressed as a percentage of the mean peak-to-peak amplitude size of the unconditioned test pulse. Mean RTs were also analyzed for each condition during the task. Reaching RTs were recorded along with the onset latency of EMG from the FDI muscle in each trial.

To clarify the relationship between FDI activity and the kinematics of the reaching movement, we performed an additional behavioral control experiment in a subset of six subjects in which MEPs were not collected, although sham TMS pulses were applied at the same delays after the acoustic cue as in the main experiment. Each subject performed two blocks of eighty trials. RTs were measured simultaneously from the deltoid muscle (EMG onset latency) and the FDI muscle (EMG onset la- 


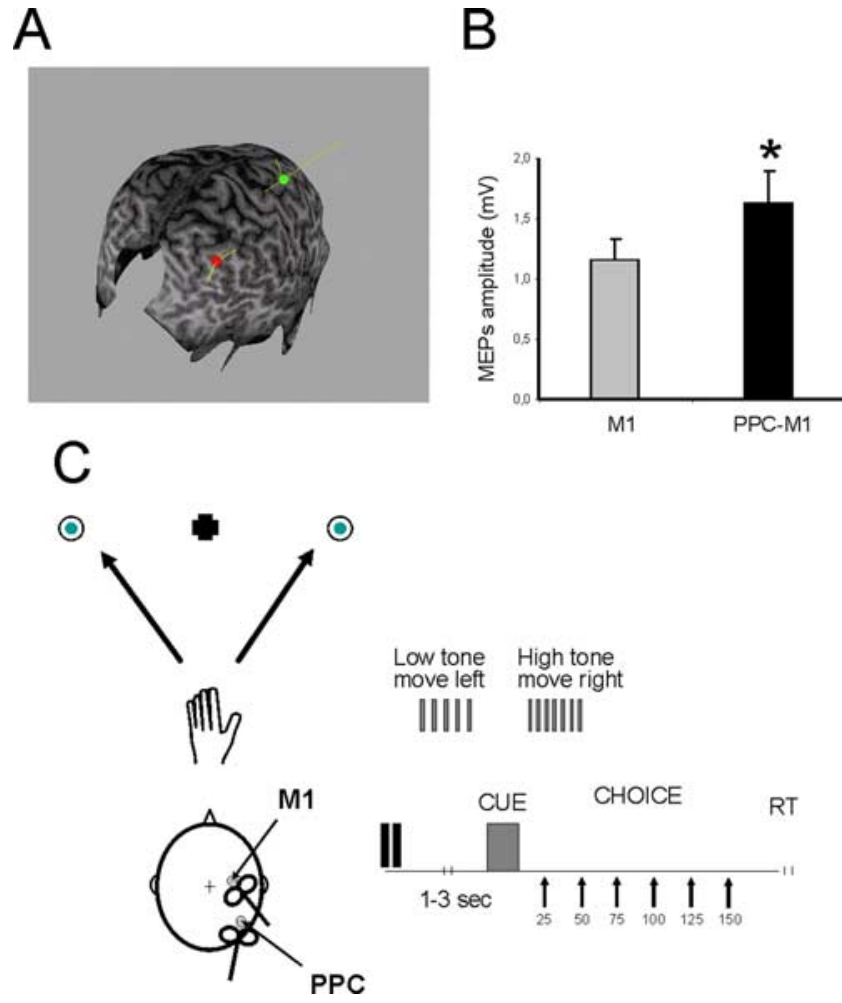

Figure 1. $A$, Conditioning TMS stimulus was applied at $90 \%$ of RMT over PPC at a site corresponding to the AG near the CIPS. B, Facilitatory effects obtained at rest after PPC conditioning in subjects of experiment 1. The intensity of test stimulus was adjusted to evoke an MEP of $\sim 1$ $\mathrm{mV}$ peak to peak in the relaxed left FDI. This M1 TMS could be preceded by a CS delivered $4 \mathrm{~ms}$ before over ipsilateral PPC at an intensity of $90 \%$ of RMT. Errors bars indicate SEM. ${ }^{*} p<0.05$.C, Schematic representation of the reaching task. At trial onset, subjects fixated the central cross. After the imperative cue sound (onsetting randomly $1-3$ s later), they reached to the left or right target with their left hand. TMS was delivered over M1 of the right hemisphere at different delays $(25,50,75,100,125$, and $150 \mathrm{~ms})$ after the cue sound onset and thus before actual reach initiation. In half of the trials, M1 TMS was preceded $4 \mathrm{~ms}$ earlier by a PPC TMS pulse (intensity of $90 \%$ RMT).

tency), and onset of hand movement was detected by release of a pushbutton. The data showed that activation of the shoulder preceded activation of FDI by just few milliseconds (for leftward movements, 271.2 vs $284.5 \mathrm{~ms}$; for rightward movements, 288.2 vs $303.2 \mathrm{~ms}$ ). Movement onset recorded with the pushbutton did not significantly differ from these values (leftward movements, $279.1 \pm 14.3$; rightward movements, $291.5 \mathrm{~ms}$ ). The onset latency of saccadic eye movements were compared using simultaneously recorded EOG and an infrared eye tracking system. For leftward saccades, mean movement onset recorded with EOG was 243.3 and $252.8 \mathrm{~ms}$ with the eye tracking device. For rightward saccades, movement onset recorded with EOG was 253.0 and $256.4 \mathrm{~ms}$ with the eye tracking device.

\section{Experiment 2}

This was similar to experiment 1 , but subjects were no longer able to see continuously the peripheral targets, because in one condition they $(n=$ 8 ) were blindfolded throughout the task while in the other they $(n=6)$ were able to see the targets only briefly just before movement onset. For the latter, we used a specific custom-made polarized screen that was positioned $10 \mathrm{~cm}$ in front of the eyes of the subject and occluded vision of the targets and of the hand. On each trial, it was activated briefly (for 100 ms) $200 \mathrm{~ms}$ before the acoustic cue to permit transient vision of the targets and of the hand immediately before a decision about the direction of reach had to be made. The purpose of this was to assess whether the reaching-dependent PPC-M1 interplay observed in experiment 1 depended on visual feedback being available for the reach target. TMS was delivered over M1 of the right hemisphere at different delays $(50,75,100$, and $125 \mathrm{~ms}$, selected based on results from experiment 1) after the cue. The intensity of M1 TMS was again adjusted to evoke an MEP of $\sim 1 \mathrm{mV}$ peak to peak in the relaxed left FDI. In half of the trials, M1 TMS was preceded by a conditioning pulse delivered $4 \mathrm{~ms}$ earlier over the ipsilateral PPC at an intensity of $90 \%$ RMT. Measurements were made as in experiment 1 . Before being blindfolded subjects performed a few practice trials to accustom themselves to the position of the peripheral targets that they had to reach toward when vision was removed. Maintained accuracy in reaching was confirmed visually by the experimenter throughout the session.

\section{Experiment 3}

This was again similar to experiment 1 , but subjects $(n=7)$ were now instructed to make a saccade toward the left or right target after the cue sound, without moving the left hand that now rested in the starting position for the whole experiment. The purpose of this was to assess whether the PPC-M1 effects observed in experiments 1 and 2, which appeared to depend on the (contralateral) direction of an upcoming planned reach, did indeed depend on reach direction in particular rather than on some nonmotoric representation of target location.

TMS was delivered over M1 of the right hemisphere at different delays (50, 75, 100, and $125 \mathrm{~ms})$ after the cue sound. The intensity of M1 TMS was again adjusted to evoke an MEP of $\sim 1 \mathrm{mV}$ peak to peak in the relaxed left FDI. In half of the trials, M1 TMS was preceded by a conditioning pulse delivered $4 \mathrm{~ms}$ before over the ipsilateral PPC at an intensity of $90 \%$ RMT. As in the previous experiments, the mean peak-to peak amplitude of the conditioned MEP was expressed as a percentage of the mean peakto-peak amplitude size of the unconditioned test pulse. Measurements were made on each individual trial. Mean saccadic latencies (derived from EOG recordings) were analyzed for each condition during the task.

\section{Experiment 4}

This experiment $(n=8)$ tested for any influences between PPC and M1 in the left hemisphere, while subjects were planning reaching movements with the right hand. (It was thus a "mirror image" of experiment 1.) TMS was delivered over M1 of the left hemisphere at different delays (25, 50, $75,100,125$, and $150 \mathrm{~ms}$ ) after the cue sound. The intensity of M1 TMS was again adjusted to evoke an $\mathrm{MEP}$ of $\sim 1 \mathrm{mV}$ peak to peak in the relaxed right FDI. In half of the trials, M1 TMS was preceded by a conditioning pulse delivered $6 \mathrm{~ms}$ earlier over the ipsilateral left PPC at an intensity of $90 \%$ RMT, because this was the most effective interstimulus interval found in our previous study to evoke facilitation in the left hemisphere (Koch et al., 2007a). The conditioning stimulus (CS) coil position for left PPC TMS was then defined relative to the P3 position of the 10-20 EEG system (Koch et al., 2007a). The coils were positioned as in experiment 1 , and equivalent measurements were obtained.

\section{Data analysis}

In experiments 1 and 4, the effects of paired stimulation over PPC, on the size of MEP recorded from the contralateral FDI in response to M1 TMS, were analyzed as the percentage of the mean peak-to-peak amplitude of the unconditioned test M1 pulse. For the reaching task, mean percentage values were analyzed with a repeated-measures ANOVA with time (25, $50,75,100,125$, and $150 \mathrm{~ms}$ ) and reaching direction (left vs right) as within-subjects factors. The same analysis was conducted on mean RTs to initiate reaches.

In experiments 2 and 3, analogous analyses were performed with time $(50,75,100$, and $125 \mathrm{~ms})$ and reaching direction (left vs right) as withinsubjects main factors. A $p$ value $<0.05$ was considered significant. A significant main effect in the ANOVA was followed by post hoc paired $t$ test analysis with Bonferroni's correction. The Greenhouse-Geisser correction for nonspherical data were used.

\section{Results}

\section{Experiment 1}

PPC-M1 $1_{\text {hand }}$ effects were first probed at rest to confirm in each subject that a CS over right cIPS at an intensity of $90 \%$ of RMT did indeed facilitate the left-hand MEP evoked by a test pulse applied $4 \mathrm{~ms}$ later over right M1. This would be expected given 
A

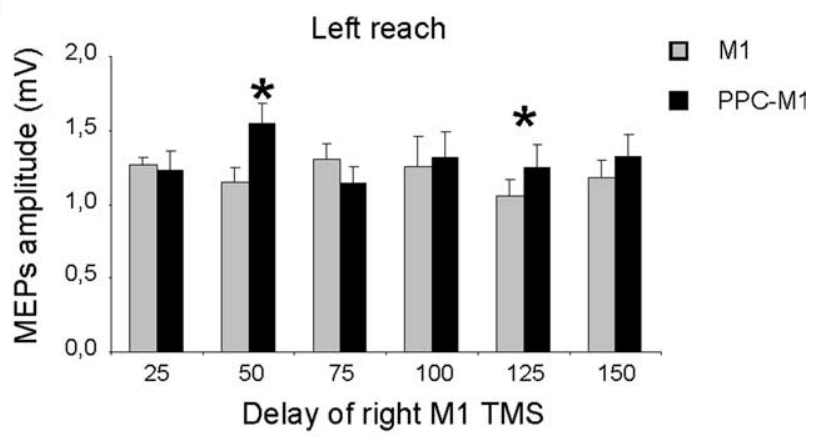

B

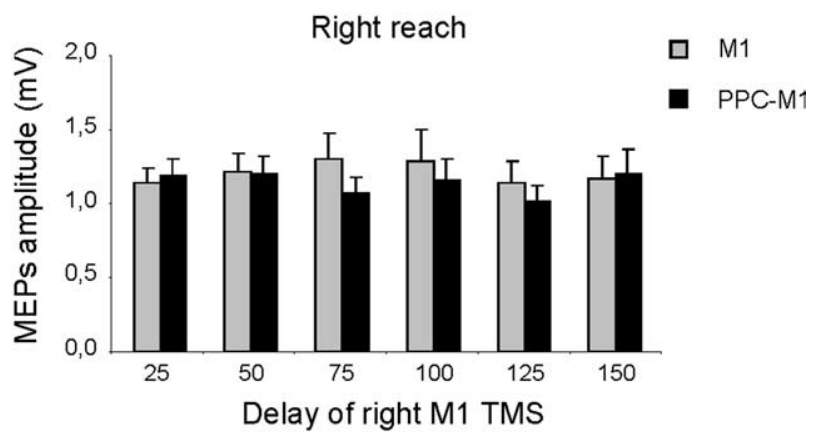

C

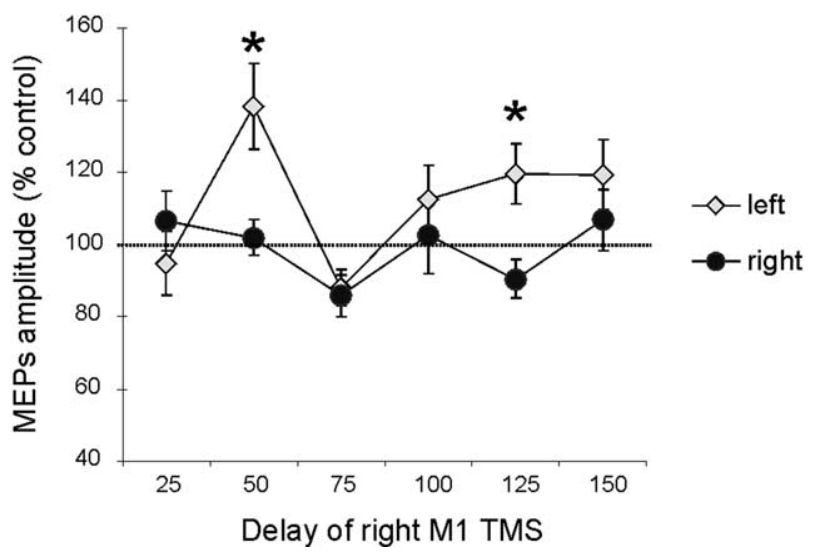

Figure 2. Effects of PPC conditioning on ipsilateral M1 excitability at different delays after the cue signal, when subjects planned left-hand reaches to visible targets on left or right. $A, B$, MEP amplitudes recorded from the left FDI during either conditions (single or paired-pulse TMS) at different time points after the imperative auditory cue, when leftward $(\boldsymbol{A})$ or rightward $(\boldsymbol{B})$ reaches were planned. Mean percentages of baseline MEP amplitude attributable to right PPC conditioning are shown in $\boldsymbol{C}$, with $100 \%$ representing no change. Corticocortical PPCM1facilitation occurred selectively at an early delay of $50 \mathrm{~ms}$ and at the later point of $125 \mathrm{~ms}$ after the auditory imperative cue for a leftward reach (yellow points in $\mathbf{C}$. There was no facilitation when a rightward reach was planned (dark points in $\mathbf{C}$. ${ }^{*} p<0.05$, post hoc analysis. Errors bars indicate SEM.

the recent findings of Koch et al. (2007a). In the present set of subjects, a CS over PPC did indeed increase MEPs by $\sim 45 \%$ $(1.15 \pm 0.65 \mathrm{mV}$ for the test pulse alone, increased to $1.63 \pm 0.78$ $\mathrm{mV}$ when a CS over PPC was applied $4 \mathrm{~ms}$ before the test pulse; $t_{(9)}=-3.11 ; p=0.01$ ) (Fig. $1 B$ ). This confirms the facilitatory functional "connectivity" or interplay between right PPC and right M1 when at rest.

Figure 2 plots the EMG data acquired during the planning phase of the reaching task, when paired-pulse TMS was applied with a fixed temporal offset $(4 \mathrm{~ms})$ between right PPC and M1, yet with a varied temporal interval between onset of the auditory signal indicating which direction to reach in, and onset of the TMS paired pulse (see $x$-axis in the plots of Fig. 2). A right PPC pulse preceded the M1 pulse on half of the trials. Figure 2, $A$ and $B$, plots left-hand MEP amplitudes, for trials with planned (but not yet initiated; see below) leftward or rightward reaches, respectively. Figure $2 C$ plots percentage facilitation of these MEPs attributable to the PPC conditioning pulse (relative to the M1only baseline), at intervals from 25 to $150 \mathrm{~ms}$ after onset of the imperative auditory signal to reach leftward or rightward with the right hand. When subjects planned a rightward reach (which on average they did not initiate until $274 \mathrm{~ms}$ after the auditory signal, i.e., considerably later than the TMS applications), the CS over PPC now had no effect on EMG response to the test M1 pulse, although facilitation had been quite clear when subjects were at rest (see above). However, when subjects planned a leftward reach instead, then facilitation attributable to the CS over PPC became evident specifically at 50 and $125 \mathrm{~ms}$ after the imperative auditory signal (although the leftward reach itself was not initiated until $261 \mathrm{~ms}$ after that auditory signal on average).

ANOVA on the percentage-facilitation data showed significant main effects of reaching direction $\left(F_{(1,9)}=5.16 ; p<0.05\right)$ and time since auditory signal $\left(F_{(1,9)}=6.54 ; p<0.005\right)$, as well as an interaction between these factors $\left(F_{(5,45)}=3.72 ; p<0.01\right)$. Post hoc analysis using Bonferroni's corrected $t$ tests showed that the PPC-M1 $1_{\text {hand }}$ reach-direction-specific facilitation arose selectively at an early delay of $50 \mathrm{~ms}$ after the auditory signal (for leftward vs rightward planned reach, $p<0.05$ ) and at a later delay of $125 \mathrm{~ms}(p<0.05)$ (Fig. $2 C$ ). This outcome was further confirmed by conducting paired $t$ tests to compare conditioned and unconditioned MEP amplitude for a given direction and time point. There was significant facilitation during planned leftward reaches at the $50 \mathrm{~ms}\left(t_{(9)}=-3.65 ; p=0.001\right)$ and $125 \mathrm{~ms}\left(t_{(9)}=\right.$ $-2.87 ; p=0.04$ ) delay (Fig. $2 A$ ), but there was no paired-pulse effect during planned rightward reaches.

Note that these changes in PPC-M1 $1_{\text {hand }}$ functional connectivity, or interplay, depended on the direction of the planned upcoming reach and on the point in time since the auditory signal. They all arose before the reach being initiated and thus unambiguously during the planning phase of the reach task. The mean RT to initiate a reach was $267 \mathrm{~ms}$ after the auditory signal (261 ms for leftward and 274 for rightward). These reach-initiation times did not vary significantly with reaching direction, nor with the timing of TMS (Tables 1,2); no terms were significant in a time $\times$ direction ANOVA. There was, however, a tendency for reaction times to be shorter when TMS pulses were given closer in time to the auditory go signal. We interpret this as a possible effect of "intersensory facilitation" between the two stimuli (sound and TMS, with the latter inevitably producing some "click" also) when given in close temporal proximity.

\section{Experiment 2}

It has been proposed previously (Mattingley et al., 1998), based on neuropsychological evidence (see Discussion), that in humans right PPC might be specifically involved in planning reaches in the contralateral leftward direction. However, as noted in Introduction, it has also been proposed that PPC might be involved in representing salient or attended visual locations.

Accordingly, in experiment 2, we tested whether the present effects between human right PPC and right M1, which were specific during the active-task conditions to contralateral reaches, were still observed when subjects were blindfolded (so that no target locations are visible). If the effect depends only on planned 
reach direction, it should presumably still be observed. If it depends instead on visual representations of target location for the upcoming reach, it might now be eliminated. Finally, because the effect in experiment 1 was observed at two different time points (Fig. 2), it is possible that reach direction (but not vision) will prove critical for one time point, whereas vision of the target location may instead become critical for the other time point.

Repeating the protocol (albeit for less points in time, given that we now knew the most effective time points) (see Materials and Methods), but with subjects now blindfolded ( $n=8$, six of whom had participated in experiment 1 ; all subjects performed a practice session without blindfold to learn the position of the targets), revealed a similar effect on inferred right PPC-M1 functional connectivity for leftward but not rightward reaches, at the early phase $(50 \mathrm{~ms})$ but no longer for the late phase $(125 \mathrm{~ms})$ of the planning period (experiment 2) (Fig. $3 A$ ). ANOVA on these data showed that, although the main effects of reaching direction and time were not significant, there was again a significant reaching direction $\times$ time interaction $\left(F_{(3,21)}=11.42 ; p<0.05\right)$. Post $h o c$ analysis using Bonferroni's corrected $t$ tests showed that facilitation by the right PPC conditioning pulse occurred selectively at the early delay of $50 \mathrm{~ms}$ (when left vs right reaches were planned, $p<0.05$ ) after the auditory imperative signal, but again only for trials on which a (contralateral) leftward reach was planned (Fig. $3 A$ ). This outcome was further confirmed by conducting paired $t$ tests on the amplitude of test and conditioned MEPs at different time intervals. There was significant facilitation when leftward reaches were planned, at the $50 \mathrm{~ms}$ delay $\left(t_{(7)}=\right.$ $-3.14 ; p=0.01)$, but there was no paired-pulse effect during planned rightward reaches.

Similar findings were obtained in a second experimental condition that used a polarizing screen to prevent vision of the targets. In this condition, subjects were able to see the targets briefly for $100 \mathrm{~ms}$ starting $200 \mathrm{~ms}$ before the acoustic cues but not at other times. ANOVA on these data showed that there was again a significant reaching direction $\times$ time interaction $\left(F_{(3,15)}=4.02\right.$; $p<0.05$ ). Post hoc analysis showed that facilitation after the right PPC conditioning pulse occurred selectively at the early delay of $50 \mathrm{~ms}$ (when left vs right reaches were planned, $p<0.05$ ) after the auditory imperative signal, but again only for trials on which a (contralateral) leftward reach was planned (Fig. $3 B$ ).

To compare directly the data obtained in the same subjects $(n=6)$ when they had to plan a leftward or rightward reach, either with vision available (experiment 1 ) or when blindfold (experiment 2), we performed a repeated-measure ANOVA on the percentage-facilitation data, with experiment and time as factors, separately for leftward-reach and rightward-reach trials. When leftward reaches were planned, in addition to the main effect of time $\left(F_{(1,5)}=3.66 ; p<0.05\right)$, the interaction of experiment $\times$ time $\left(F_{(3,15)}=7.52 ; p<0.05\right)$ was also significant. Post hoc analysis using Bonferroni's corrected $t$ tests confirmed that, when left reaches were planned, the two experiments differed in their outcome only at the late $125 \mathrm{~ms}$ delay $(p<0.05)$ after the imperative signal (Fig. 4). At the $50 \mathrm{~ms}$ delay, the same facilitatory effect was observed when both sighted and blindfolded. There were no significant differences between experiments when rightward reaches were planned (no facilitation attributable to PPC TMS being found at any delay when rightward reaches were planned).

\section{Experiment 3}

The results for blindfolded subjects in experiment 2 suggested that the reach-direction-specific functional connectivity between PPC and M1, as found at the early $50 \mathrm{~ms}$ delay, does not reflect vision of the target location for the upcoming reach, because it was replicated in blindfolded subjects planning spatial reaches without vision of the target location. In contrast, at the later 125 ms delay, vision of the target location for the upcoming reach may evidently play some role, because the outcome at this time point differed significantly between experiments 1 and 2 (with PPC-M $1_{\text {hand }}$ facilitation being eliminated by the blindfold at the later, but not the earlier, delay). Our next experiment sought additional evidence that the reach-direction-dependent functional connectivity between PPC and M1 is indeed specific to reaches rather than to spatial selection of a motor plan more generally. To address this, we now required subjects (without a blindfold) to make leftward or rightward saccades after the auditory imperative signal rather than reaches (for a somewhat analogous logic, although using the very different method of invasive monkey neurophysiology in PPC) (Snyder et al., 1997). Subjects $(n=7$, five of whom participated in experiments 1 and 2) no longer had to prepare any reach movements.

The conditioning right PPC pulse no longer had any effect when subjects planned saccades rather than reaches (Fig. 5A,B). An ANOVA on these data showed that only the main effect of time was significant $\left(F_{(1,6)}=4.51 ; p<0.05\right)$, with no effect or interaction involving planned saccade direction (Fig. 5A,B). Analysis of saccade latencies did not show any significant effects of condition (Table 1) and also confirmed that TMS was always applied before saccade execution (as had also been the case for reaches).

To compare directly the data obtained when the same nonblindfolded subjects $(n=5)$ planned a leftward reach versus a saccade, we performed a repeated-measure ANOVA on the percentage-facilitation data (Fig. $5 C$ ), with task (hand/eye, equivalent to experiment 1 vs experiment 3 ) and time as factors, for 
A

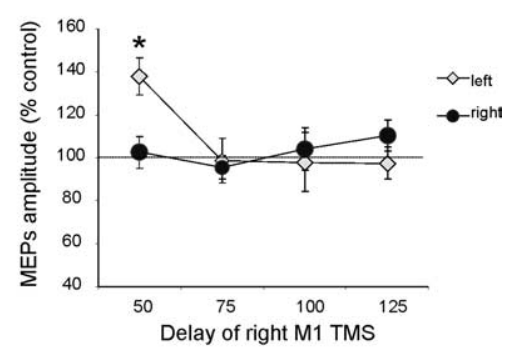

B

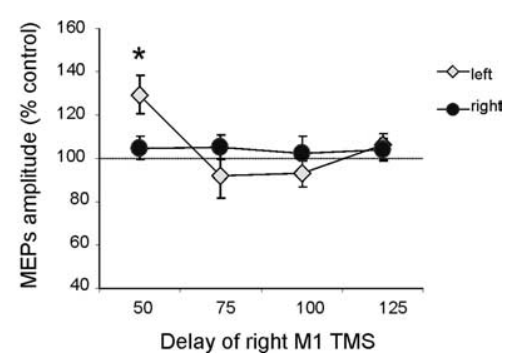

Figure 3. $P P C-M 1$ functional connectivity during movement planning in space with subjects blindfolded $(\boldsymbol{A})$ or when subjects were allowed to see briefly the targets before the acoustic cues $(\boldsymbol{B})$. When subjects were not allowed to see peripheral targets $(\boldsymbol{A})$, we found a similar profile of activation of $\mathrm{PPC}-\mathrm{M} 1$ functional connectivity during the early phase of movement planning. Post hoc analysis showed that corticocortical facilitation using a conditioning intensity of $90 \%$ RMT with 4 ms interstimulus interval occurred selectively at an early delay of $50 \mathrm{~ms}$ after the auditory imperative signal, only for trials in which a contralateral left reach was reached. This pattern of activation of PPC-M1 connectivity was the same when subjects were allowed to see transiently the targets before they were asked to decide where to reach $(\boldsymbol{B})$. Values are expressed as mean percentage for MEP amplitude attributable to right PPC conditioning. ${ }^{*} p<0.05$, post hoc analysis. Errors bars indicate SEM.

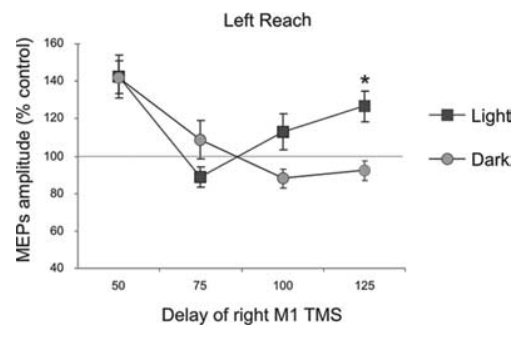

Figure 4. Comparison between results obtained in experiments 1 and 2 for the common delays, comparing the results obtained when subjects were planning a leftward movement with vision (Light) or when blindfolded (Dark). A late peak of facilitation at $125 \mathrm{~ms}$ for the $\mathrm{PPC}-\mathrm{M} 1_{\text {hand }}$ interaction was evident only when subjects were able to see the peripheral targets. ${ }^{*} p<0.05$, post hoc analysis. Errors bars indicate SEM. Note that significant facilitation (relative to the $100 \%$ baseline) was observed for both the light and the blindfolded condition at the early 50 ms delay.

data from the common delays of experiments 1 and 3 (i.e., for 50, 75,100 , and $125 \mathrm{~ms}$ after the auditory imperative signal). This revealed a significant task $\times$ time interaction $\left(F_{(3,12)}=9.32 ; p<\right.$ $0.001)$. Facilitation was significantly larger $(p<0.05$ using Bonferroni's corrected $t$ tests) at the 50 and at the $125 \mathrm{~ms}$ delay for planned leftward hand movements compared with planned leftward saccades (Fig. $5 C$ ). Thus, the early PPC-M1 hand functional connectivity found during the early stages of planning a leftward reach (as in both experiments 1 and 2), at the $50 \mathrm{~ms}$ interval, was not found when planning a leftward saccade instead, under otherwise comparable conditions. Analysis for trials with planned rightward movements did not find any difference between planned reaches and saccades for the comparable delays of exper-
A

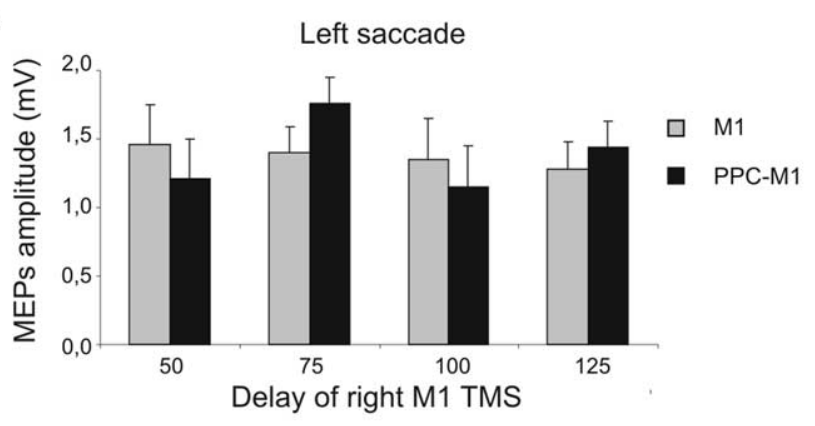

B

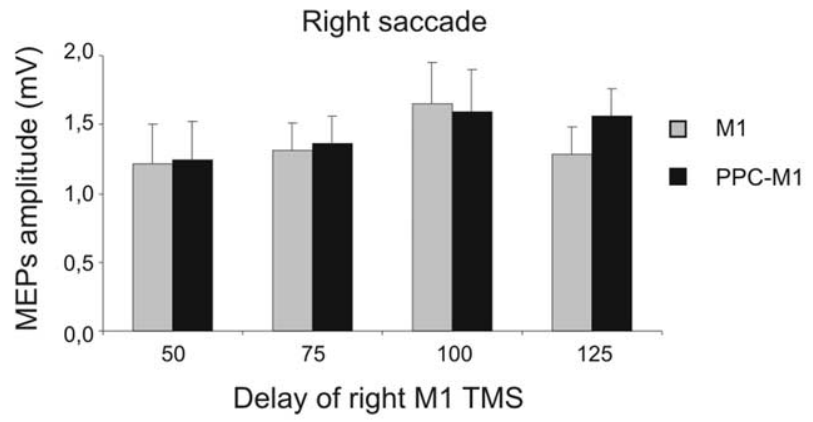

C

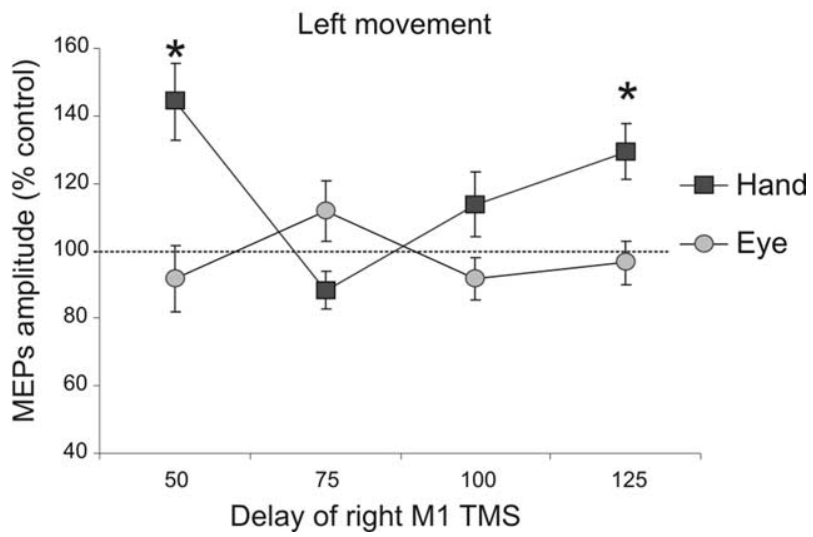

Figure 5. $\quad P P C-M 1$ functional connectivity is absent during saccade planning (experiment 3). $P P C-M 1$ effects were not evident during a saccadic task. Unlike the reach tasks (experiments 1 and 2), there was now no significant facilitation at any time point. $\boldsymbol{A}, \boldsymbol{B}$, MEP amplitude recorded from the left FDI during either condition (single or paired-pulse TMS) at different time points when leftward $(\boldsymbol{A})$ or rightward $(\boldsymbol{B})$ saccades were planned. $\boldsymbol{C}$, Normalized data (mean percentage values of MEP amplitude during right PPC conditioning, with $100 \%$ indicating no effect) obtained in experiments 1 and 3 , comparing the results obtained when subjects were planning a leftward movement with either the hand or the eyes, respectively. The early peak of facilitation at $50 \mathrm{~ms}$ for the PPC $-\mathrm{M1}_{\text {hand }}$ interaction was evident only when subjects were planning reaching movements toward the contralateral left side but not for planned saccades toward that side. ${ }^{*} p<0.05$, post hoc analysis. Errors bars indicate SEM.

iments 1 and 3 (i.e., no paired-pulse facilitation was found in any condition where a rightward movement was planned; see also experiment 2).

\section{Experiment 4}

This experiment now tested for $\mathrm{PPC}-\mathrm{M} 1_{\text {hand }}$ effects within the left hemisphere instead, during the context of a reaching task analogous to experiment 1 . Our previous paper, which examined PPC-M1 effects only during rest (Koch et al., 2007a), had already showed that a conditioning pulse over left PPC can facilitate ipsilateral M1 at rest, with a maximal effect for an interpulse delay of $6 \mathrm{~ms}$, so this timing was used here. The present experiment 4 
was similar to experiment 1 , except that, in this group of subjects ( $n=8$, six of whom participated in experiment 1 ), left M1 TMS could now be preceded by a CS delivered $6 \mathrm{~ms}$ earlier, over left PPC at an intensity of $90 \%$ RMT. For the reaching task, subjects were now required to move the right hand instead of the left hand, thus making experiment 4 a mirror-image analog of experiment 1 . At rest in the present sample, we observed facilitation of MEPs after a conditioning left PPC pulse $(1.07 \pm 0.32$ vs $1.36 \pm$ $\left.0.61 \mathrm{mV} ; t_{(7)}=-3.01 ; p=0.01\right)$. These effects observed at rest for left PPC-M1 interplay are somewhat milder in term of relative facilitation compared with those evoked in the right hemisphere (for similar findings at rest) (Koch et al., 2007a).

ANOVA on the percentage-facilitation data during the reach task with the right hand showed significant main effects of reaching direction $\left(F_{(1,7)}=3.41 ; p<0.05\right)$ and time since auditory signal $\left(F_{(1,7)}=4.08 ; p<0.05\right)$, as well as an interaction between these factors $(\mathrm{F}(5,35)=2.94 ; p<0.05)$. Post hoc analysis using Bonferroni's corrected $t$ tests showed that the PPC-M1 reachdirection-specific facilitation arose selectively at an early delay of $50 \mathrm{~ms}$ after the auditory signal (for rightward vs leftward planned reach, $p<0.05)$ and at a later delay of $100 \mathrm{~ms}(p<0.05)$ (Fig. $6 C)$. This outcome was further confirmed by conducting paired $t$ tests to compare conditioned and unconditioned MEP amplitude for a given direction and time point. There was significant facilitation during planned rightward reaches at the $50 \mathrm{~ms}\left(t_{(7)}=\right.$ $-2.95 ; p=0.02)$ and $100 \mathrm{~ms}\left(t_{(7)}=-2.62 ; p=0.03\right)$ delay (Fig. $6 B)$, but there was no paired-pulse effect during planned leftward reaches (Fig. 6A). This therefore shows a similar (mirror-image) dependency on reach planning in the contralateral direction as for the right-hemisphere results in the previous experiments in this paper.

To summarize, the present experiments show for the first time that paired-pulse TMS effects between PPC and $M 1_{\text {hand }}$ in humans, as reported recently at rest (Koch et al., 2007a), can in fact be highly dependent on task conditions and in particular on current motor state. If subjects were at rest, a conditioning right PPC pulse consistently facilitated EMG responses evoked by a subsequent right M1 test pulse when the interval between pulses was 4 ms (Koch et al., 2007a). However, this influence from PPC was eliminated during several task conditions in the present experiments but specifically present during other active conditions. In particular, when preparing an (ipsilateral) rightward reach, or a saccade toward either the left or right, no facilitatory paired-pulse effects were observed for right PPC-M1 hand TMS. However, when preparing a (contralateral) leftward reach instead, a reliable facilitatory effect was found at both an early $(50 \mathrm{~ms})$ and later (125 ms) time point after the auditory imperative signal to execute the reach (but before actual initiation of that movement). Similar results were observed when testing for PPC-M1 effects within the left hemisphere. Again during active task conditions, facilitation arose at specific time points only when subjects were planning a reach toward the contralateral space (now rightward), not when planning an ipsilateral reach.

\section{Discussion}

We suggest that the time-varying, task-dependent and conditiondependent functional connectivity described here for the first time reflects a real-time contribution of PPC in initial programming of reach movements that influences M1.

Previous studies in nonhuman primates have suggested that PPC may be involved in converting spatial specification of target location into motor intentions (for review, see Cohen and Andersen, 2002). It has also been proposed (Cavada and
A

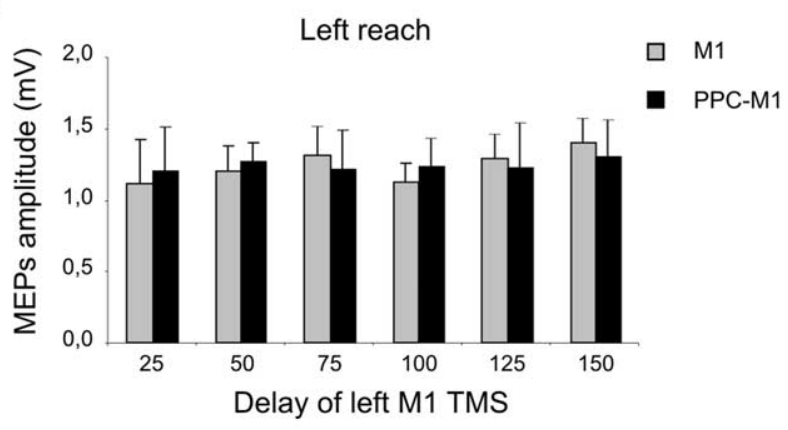

B

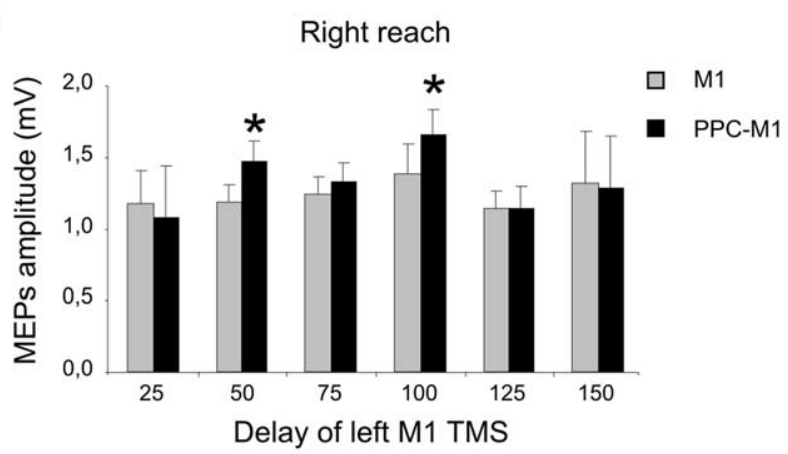

C

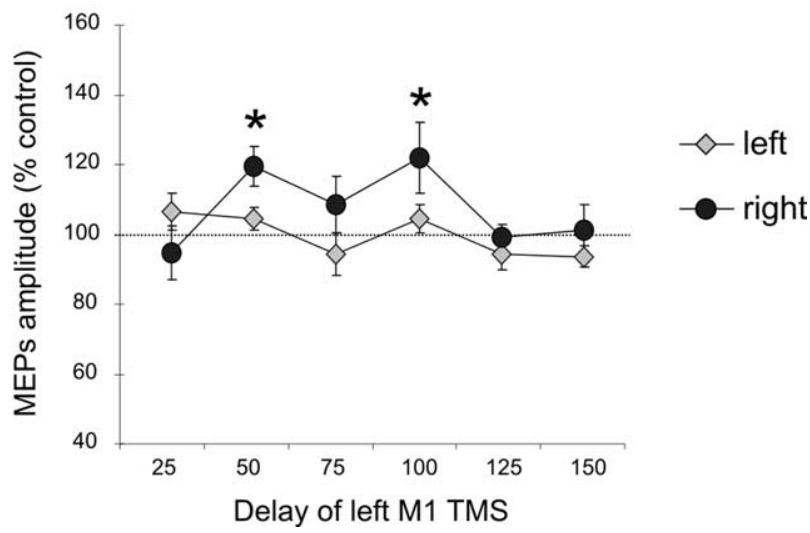

Figure 6. Experiment 4: effects of left PPC conditioning on ipsilateral M1 excitability at different delays after the cue signal, when subjects planned right-hand reaches to visible targets on left or right. $A, B$, MEP amplitudes recorded from the right FDI during either conditions (single or paired-pulse TMS) at different time points after the imperative auditory cue, when leftward $(\boldsymbol{A})$ or rightward $(\boldsymbol{B})$ reaches were planned. Mean percentages of baseline MEP amplitude attributable to right PPC conditioning are shown in $C$, with $100 \%$ representing no change. Corticocortical PPC-M1 facilitation occurred selectively at an early delay of 50 and $100 \mathrm{~ms}$ after the auditory imperative cue for a contralateral rightward reach (dark points in $\mathbf{C}$. There was no facilitation when a leftward reach was planned instead (light gray points in $C$ ). ${ }^{*} p<0.05$, post hoc analysis. Errors bars indicate SEM.

Goldman-Rakic, 1989; Seltzer and Pandya, 1994; Johnson et al., 1996; Snyder et al., 1997) that, within PPC, nascent motor intentions may be specific to one class of movement (e.g., reaching) versus another (e.g., saccades), as also found for the new tcTMS phenomena uncovered here in humans. Moreover, parietal regions may over-represent the contralateral workspace relative to the ipsilateral workspace (Battaglia-Mayer et al., 2005). Damage to such representations in human patients might explain some directional motor aspects of neglect, whereby for instance parietal patients (whose damage would include the site targeted with right PPC TMS in normals here) can be impaired at initiating 
reaches in the contralesional direction, over and above any visual or attentional impairments for targets on that side (Heilman et al., 1985; Mattingley et al., 1998; Husain et al., 2000). A role for PPC in reaching may also be broadly consistent with the frank misreaching deficits in monkeys (Lamotte and Acuña, 1978) and in patients with optic ataxia (Ratcliff and Davis-Jones, 1978; Jeannerod, 1986), whose lesions can include not only medial occipitoparietal junction, but also the superior occipital gyrus, the IPS, the superior parietal lobule, and the IPL, the latter particularly on the right (Karnath and Perenin, 2005).

Reaching (with some arm transport, as opposed to just hand/ finger movements) has been relatively difficult to investigate with function MRI (fMRI) because of technical aspects such as head motion induced when reaching or MR artifacts from the moving arm (Culham et al., 2006). Those fMRI investigations of reaching that overcame such issues typically reported fairly widespread cortical activations involving dorsal premotor cortex as well as superior parietal lobe and IPS. Along the IPS, activation was found mainly at the medial IPS (Grefkes et al., 2002; Prado et al., 2005), although some studies showed a broader activation extending to cIPS (Filimon et al., 2007), the PPC site we explored in the current study. Behavioral TMS investigations (not using the tcTMS methodology introduced here) showed that more rostral sites, such as the anterior IPS, seem to be involved mostly in grasping preparation (Tunik et al., 2005; Rice et al., 2006; Davare et al., 2007) whereas more posterior sites, such as medial IPS (Desmurget et al., 1999) and cIPS as in the present study), may potentially contribute to reaching movements in space.

Studies in monkeys have suggested that the main cortical circuits underlying reaching involve the superior parietal lobule and PMd-MI (Johnson et al., 1996; Marconi et al., 2001) with weaker connectivity between IPL areas and PMd or MI (Pandya and Seltzer, 1982; Rozzi et al., 2006). However, it is possible that in humans a more prominent role is played by other circuits, including the one tested in the current study (Pisella et al., 2006). Furthermore, comparison with these different fields of research and with fMRI results remain rather speculative, because different task conditions have been used and anatomical data sometimes are difficult to compare. In particular, we cannot rule out that cIPS stimulation could partially propagate to regions that are activated by reaching in humans using fMRI such as the medial IPS situated laterally and the parietooccipital junction located medially.

In the present paradigm, subjects had foreknowledge of the two possible target locations throughout all the active task conditions. The imperative auditory tone thus did not provide new spatial information but instead indicated symbolically which of the two reach movements to execute. When the upcoming reach was contralateral (e.g., leftward when using right TMS sites), there was an initial facilitation of $\mathrm{PPC}-\mathrm{M} 1_{\text {hand }}$ functional effects, $50 \mathrm{~ms}$ after the tone. We suggest that this early facilitation reflects initial biasing of movement plans in the contralateral direction, which may then shape motor cortex excitability and motor engrams dedicated for performing leftward reaching movements. Although this peak of facilitatory functional connectivity appeared remarkably early [potentially consistent with suggestions that parietal contributions to motor planning arise at the earliest stages of nascent motor intention, (Cohen and Andersen, 2002)], this falls within the temporal range of a recent magnetoencephalography study that showed that auditory signals can arrive in PPC within $<40 \mathrm{~ms}$ (Inui et al., 2006).

The early peak of facilitatory functional effects for right PPC$\mathrm{M} 1_{\text {hand }}$, in the context of planning a contralateral leftward reach, was present even in blindfolded subjects or when the experimen- tal scenario was presented transiently (experiment 2). This may accord with other work showing involvement of PPC during reaching tasks even when executed in darkness (Battaglia-Mayer et al., 2000, 2003). An additional key aspect of our findings was that PPC-M1 $1_{\text {hand }}$ effects were not evident when planning directional saccades rather than directional reaches, in an otherwise comparable paradigm (experiment 3 ). This indicates that the PPC-M1 circuit tapped into here may specifically be involved in control of hand movements, rather than in spatial planning of movements regardless of effector, or in spatial attention more generally. It should be interesting in future work to test for any analogous circuits in the human brain specific to saccades rather than reaches (e.g., for functional connectivity between other parietal regions and frontal eye fields), as might be addressed with the new methodological combination of concurrent TMS-fMRI (Bestmann et al., 2005; Ruff et al., 2006).

In addition to the early peak in excitability of right PPC$M 1_{\text {hand }}$ when a contralateral leftward reach was planned, at a delay of $50 \mathrm{~ms}$, there was also a later peak at $125 \mathrm{~ms}$ that only occurred when subjects were able to see the target (experiment 1) but not when blindfolded or when they could see the stimuli only briefly (experiment 2). The dependence of our later PPC-M1 facilitatory effect on vision of target locations may reflect a recursive cycle, in which initial motor intentions then lead to reliance on visual information (Ferraina et al., 1997; Prado et al., 2005; Clavagnier et al., 2007). Availability of visual input at this later stage of processing may be used by PPC-M1 circuits to define the exact location of the peripheral visual target more precisely. This could accord with the general idea that PPC is important for integrating visual and motoric information (Cohen and Andersen, 2002). More specifically, we can now propose that the early peak of PPC-M1 facilitation at $50 \mathrm{~ms}$ may reflect the directional aspect of reaching movements (i.e., intention to move toward contralateral space), not necessary goal-directed, because it is still present when subjects blindfolded perform stereotyped pantomime reaching movements in one or the other direction, whereas the later peak at $125 \mathrm{~ms}$ that depends on visual input may contribute to the spatial accuracy of motor planning.

Although significant facilitation occurred in the early phase of the task, and a vision-dependent effect later, the absence of effects at other (intermediate) time points indicates high temporal specificity in PPC-M1 interplay, as we replicated here several times. The effective time points presumably correspond to peaks in excitability of interconnections. Interestingly, a rather similar profile of transient task-dependent activation and suppression of corticocortical connections was observed in recent studies exploring the role of PMd-contralateral M1 connectivity during movement selection with auditory (Koch et al., 2006) or visual cues (Boorman et al., 2007). Such processes of temporally specific activation or suppression of different corticocortical pathways, involved in distinct motor behaviors, may thus be a common feature of cortical information processing.

We did not assess here whether the observed specific changes in right $\mathrm{PPC}-\mathrm{M} 1_{\text {hand }}$ interplay during contralateral leftward reach planning depend specifically on modulation of intracortical circuits within primary motor cortex itself. However, several previous studies clearly show that such changes within M1 only arise just before the onset of actual movements (Reynolds and Ashby, 1999), not at early stages of planning (Koch et al., 2006). Therefore, it seems unlikely that changes in, say, short interval intracortical inhibition (Kujirai et al., 1993) could account for the highly specific pattern of PPC-M1 connectivity found in the current study. 
Finally, we observed that the PPC-M1 $1_{\text {hand }}$ effects showed similar profiles within either hemisphere, with facilitation rising selectively during planning of reach movements toward the contralateral space in either case, at an early and later time point, with no effect at intermediate times. However, a slight shift in time was observed for the later peak of activation when PPC-M $1_{\text {hand }}$ connectivity was tested in the left hemisphere. Whereas the first peak occurred at the same delay (50 ms) for either hemisphere, the second peak of facilitation was evident in the left hemisphere after $100 \mathrm{~ms}$ rather than at $125 \mathrm{~ms}$ as for the right hemisphere. One possibility is that the slight time shift for the later effect might reflect asymmetries in speed of visual processing between the two hemisphere, given that (as experiment 2 showed) the later effect depends on visual input being available.

In conclusion, our findings use a non-invasive method in humans to show for the first time that functional interplay between PPC and M1 is not fixed but can change in a highly task-, condition-, and time-dependent manner during the planning phase of a reaching task. cIPS can exert early influences on M1 that are facilitatory in the context of planning a reach to a contralateral target, with an initial phase that does not depend on vision of targets and a later phase that does. This PPC-M1 interplay thus relates to rapid planning of contralateral reaches.

\section{References}

Andersen RA, Buneo CA (2002) Intentional maps in posterior parietal cortex. Annu Rev Neurosci 25:189-220.

Battaglia-Mayer A, Ferraina S, Mitsuda T, Marconi B, Genovesio A, Onorati P, Lacquaniti F, Caminiti R (2000) Early coding of reaching in the parietooccipital cortex. J Neurophysiol 83:2374-2391.

Battaglia-Mayer A, Caminiti R, Lacquaniti F, Zago M (2003) Multiple levels of representation of reaching in the parieto-frontal network. Cereb Cortex 13:1009-1022.

Battaglia-Mayer A, Mascaro M, Brunamonti E, Caminiti R (2005) The overrepresentation of contralateral space in parietal cortex: a positive image of directional motor components of neglect? Cereb Cortex 15:514-525.

Baumer T, Bock F, Koch G, Lange R, Rothwell JC, Siebner HR, Munchau A (2006) Magnetic stimulation of human premotor or motor cortex produces interhemispheric facilitation through distinct pathways. J Physiol (Lond) 572:857-868.

Bestmann S, Baudewig J, Siebner HR, Rothwell JC, Frahm J (2005) BOLD MRI responses to repetitive TMS over human dorsal premotor cortex. NeuroImage 28:22-29.

Boorman ED, O'Shea J, Sebastian C, Rushworth MF, Johansen-Berg H (2007) Individual differences in white-matter microstructure reflect variation in functional connectivity during choice. Curr Biol 17:1426-1431.

Buneo CA, Jarvis MR, Batista AP, Andersen RA (2002) Direct visuomotor transformations for reaching. Nature 416:632-636.

Caminiti R, Ferraina S, Johnson PB (1996) The sources of visual information to the primate frontal lobe: a novel role for the superior parietal lobule. Cereb Cortex 6:319-328.

Cavada C, Goldman-Rakic PS (1989) Posterior parietal cortex in rhesus monkey. I. Parcellation of areas based on distinctive limbic and sensory corticocortical connections. J Comp Neurol 287:393-421.

Civardi C, Cantello R, Asselman P, Rothwell JC (2001) Transcranial magnetic stimulation can be used to test connections to primary motor areas from frontal and medial cortex in humans. NeuroImage 14:1444-1453.

Clavagnier S, Prado J, Kennedy H, Perenin MT (2007) How humans reach: distinct cortical systems for central and peripheral vision. The Neuroscientist 13:22-27.

Cohen YE, Andersen RA (2002) A common reference frame for movement plans in the posterior parietal cortex. Nat Rev Neurosci 3:553-562.

Colby CL, Goldberg ME (1999) Space and attention in parietal cortex. Annu Rev Neurosci 22:319-349.

Croxson PL, Johansen-Berg H, Behrens TE, Robson MD, Pinsk MA, Gross CG, Richter W, Richter MC, Kastner S, Rushworth MF (2005) Quantitative investigation of connections of the prefrontal cortex in the human and macaque using probabilistic diffusion tractography. J Neurosci 25:8854-8866.

Culham JC, Cavina-Pratesi C, Singhal A (2006) The role of parietal cortex in visuomotor control: what have we learned from neuroimaging? Neuropsychologia 44:2668-2684.

Davare M, Andres M, Clerget E, Thonnard JL, Olivier E (2007) Temporal dissociation between hand shaping and grip force scaling in the anterior intraparietal area. J Neurosci 27:3974-3980.

Desmurget M, Epstein CM, Turner RS, Prablanc C, Alexander GE, Grafton ST (1999) Role of the posterior parietal cortex in updating reaching movements to a visual target. Nat Neurosci 2:563-567.

Ferbert A, Priori A, Rothwell JC, Day BL, Colebatch JG, Marsden CD (1992) Interhemispheric inhibition of the human motor cortex. J Physiol (Lond) 453:525-546.

Ferraina S, Garasto MR, Battaglia-Mayer A, Ferraresi P, Johnson PB, Lacquaniti F, Caminiti R (1997) Visual control of hand-reaching movement: activity in parietal area 7m. Eur J Neurosci 9:1090-1095.

Filimon F, Nelson JD, Hagler DJ, Sereno MI (2007) Human cortical representations for reaching: mirror neurons for execution, observation, and imagery. NeuroImage 37:1315-1328.

Grefkes C, Weiss PH, Zilles K, Fink GR (2002) Crossmodal processing of object features in human anterior intraparietal cortex: an fMRI study implies equivalencies between humans and monkeys. Neuron 35:173-184.

Heilman KM, Bowers D, Coslett HB, Whelan H, Watson RT (1985) Directional hypokinesia: prolonged reaction times for leftward movements in patients with right hemisphere lesions and neglect. Neurology $35: 855-859$.

Herwig U, Satrapi P, Schonfeldt-Lecuona C (2003) Using the international 10-20 EEG system for positioning of transcranial magnetic stimulation. Brain Topogr 16:95-99.

Husain M, Mattingley JB, Rorden C, Kennard C, Driver J (2000) Distinguishing sensory and motor biases in parietal and frontal neglect. Brain 123:1643-1659.

Inui K, Okamoto H, Miki K, Gunji A, Kakigi R (2006) Serial and parallel processing in the human auditory cortex: a magnetoencephalographic study. Cereb Cortex 16:18-30.

Jeannerod M (1986) Mechanisms of visuomotor coordination: a study in normal and brain-damaged subjects. Neuropsychologia 24:41-78.

Johnson PB, Ferraina S, Bianchi L, Caminiti R (1996) Cortical networks for visual reaching: physiological and anatomical organization of frontal and parietal lobe arm regions. Cereb Cortex 6:102-119.

Kalaska JF, Crammond DJ (1995) Deciding not to GO: neuronal correlates of response selection in a GO/NOGO task in primate premotor and parietal cortex. Cereb Cortex 5:410-428.

Kalaska JF, Cohen DA, Prud'homme M, Hyde ML (1990) Parietal area 5 neuronal activity encodes movement kinematics, not movement dynamics. Exp Brain Res 80:351-364.

Karnath HO, Perenin MT (2005) Cortical control of visually guided reaching: evidence from patients with optic ataxia. Cereb Cortex 15:1561-1569.

Koch G, Franca M, Fernandez Del Olmo M, Cheeran B, Milton R, Alvarez Sauco M, Rothwell JC (2006) Time course of functional connectivity between dorsal premotor and contralateral motor cortex during movement selection. J Neurosci 26:7452-7459.

Koch G, Fernandez Del Olmo M, Cheeran B, Ruge D, Schippling S, Caltagirone C, Rothwell JC (2007a) Focal stimulation of the posterior parietal cortex increases the excitability of the ipsilateral motor cortex. J Neurosci 27:6815-6822.

Koch G, Franca M, Mochizuki H, Marconi B, Caltagirone C, Rothwell JC (2007b) Interactions between pairs of transcranial magnetic stimuli over the human left dorsal premotor cortex differ from those seen in primary motor cortex. J Physiol (Lond) 578:551-562.

Kujirai T, Caramia MD, Rothwell JC, Day BL, Thompson PD, Ferbert A, Wroe S, Asselman P, Marsden CD (1993) Corticocortical inhibition in human motor cortex. J Physiol (Lond) 471:501-519.

Lacquaniti F, Guigon E, Bianchi L, Ferraina S, Caminiti R (1995) Representing spatial information for limb movement: role of area 5 in the monkey. Cereb Cortex 5:391-409.

Lamotte RH, Acuña C (1978) Defects in accuracy of reaching after removal of posterior parietal cortex in monkeys. Brain Res 139:309-326.

Makris N, Kennedy DN, McInerney S, Sorensen AG, Wang R, Caviness Jr VS, Pandya DN (2005) Segmentation of subcomponents within the supe- 
rior longitudinal fascicle in humans: a quantitative, in vivo, DT-MRI study. Cereb Cortex 15:854-869.

Marconi B, Genovesio A, Battaglia-Mayer A, Ferraina S, Squatrito S, Molinari M, Lacquaniti F, Caminiti R (2001) Eye-hand coordination during reaching. I. Anatomical relationships between parietal and frontal cortex. Cereb Cortex 11:513-527.

Mattingley JB, Husain M, Rorden C, Kennard C, Driver J (1998) Motor role of human inferior parietal lobe revealed in unilateral neglect patients. Nature 392:179-182.

Mochizuki H, Huang YZ, Rothwell JC (2004) Interhemispheric interaction between human dorsal premotor and contralateral primary motor cortex. J Physiol (Lond) 561:331-338.

Mountcastle VB (1995) The parietal system and some higher brain functions. Cereb Cortex 5:377-390.

Mountcastle VB, Lynch JC, Georgopoulos A, Sakata H, Acuna C (1975) Posterior parietal association cortex of the monkey: command functions for operations within extrapersonal space. J Neurophysiol 38:871-908.

Pandya DN, Seltzer B (1982) Intrinsic connections and architectonics of posterior parietal cortex in the rhesus monkey. J Comp Neurol 204:196-210.

Pisella L, Binkofski F, Lasek K, Toni I, Rossetti Y (2006) No doubledissociation between optic ataxia and visual agnosia: multiple substreams for multiple visuo-manual integrations. Neuropsychologia 44:2734-2748.

Prado J, Clavagnier S, Otzenberger H, Scheiber C, Kennedy H, Perenin MT (2005) Two cortical systems for reaching in central and peripheral vision. Neuron 48:849-858.

Ratcliff G, Davies-Jones GA (1972) Defective visual localization in focal brain wounds. Brain 95:49-60.
Reynolds C, Ashby P (1999) Inhibition in the human motor cortex is reduced just before a voluntary contraction. Neurology 53:730-735.

Rice NJ, Tunik E, Grafton ST (2006) The anterior intraparietal sulcus mediates grasp execution, independent of requirement to update: new in sights from transcranial magnetic stimulation. J Neurosci 26:8176-8182.

Rozzi S, Calzavara R, Belmalih A, Borra E, Gregoriou GG, Matelli M, Luppino G (2006) Cortical connections of the inferior parietal cortical convexity of the macaque monkey. Cereb Cortex 16:1389-1417.

Ruff CC, Blankenburg F, Bjoertomt O, Bestmann S, Freeman E, Haynes JD, Rees G, Josephs O, Deichmann R, Driver J (2006) Concurrent TMSfMRI and psychophysics reveal frontal influences on human retinotopic visual cortex. Curr Biol 16:1479-1488.

Rushworth MF, Taylor PC (2006) TMS in the parietal cortex: updating representations for attention and action. Neuropsychologia 44:2700-2716.

Rushworth MF, Behrens TE, Johansen-Berg H (2006) Connection patterns distinguish 3 regions of human parietal cortex. Cereb Cortex 16:1418-1430.

Seltzer B, Pandya DN (1994) Parietal, temporal, and occipital projections to cortex of the superior temporal sulcus in the rhesus monkey: a retrograde tracer study. J Comp Neurol 343:445-463.

Snyder LH, Batista AP, Andersen RA (1997) Coding of intention in the posterior parietal cortex. Nature 386:167-170.

Tanne-Gariepy J, Rouiller EM, Boussaoud D (2002) Parietal inputs to dorsal versus ventral premotor areas in the macaque monkey: evidence for largely segregated visuomotor pathways. Exp Brain Res 145:91-103.

Tunik E, Frey SH, Grafton ST (2005) Virtual lesions of the anterior intraparietal area disrupt goal-dependent on-line adjustments of grasp. Nat Neurosci 8:505-511. 